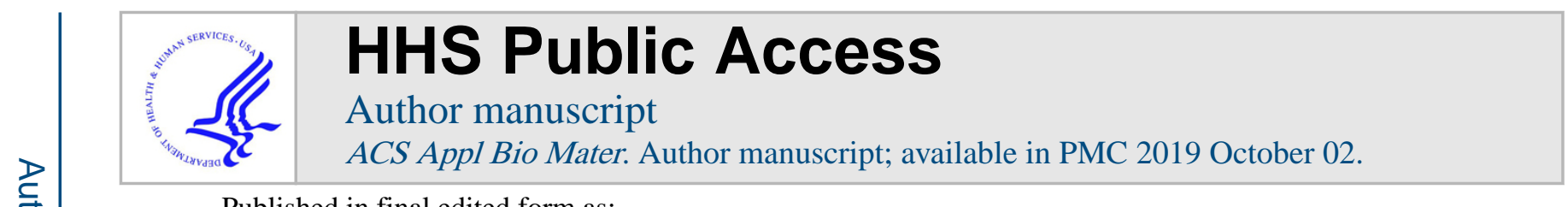

Published in final edited form as:

ACS Appl Bio Mater. 2019 September 16; 2(9): 3926-3933. doi:10.1021/acsabm.9b00502.

\title{
Bacteria-Resistant, Transparent, Free-Standing Films Prepared from Complex Coacervates
}

\author{
Irene S. Kurtz ${ }^{\dagger}$, Shuo Sui ${ }^{\dagger}, \mathrm{Xi} \mathrm{Hao}^{\dagger}$, Mengfei Huang, Sarah L. Perry ${ }^{\star}$, Jessica D. Schiffman ${ }^{\star}$ \\ Department of Chemical Engineering, Institute of Applied Life Sciences, University of \\ Massachusetts Amherst, Amherst, Massachusetts 01003, United States
}

\begin{abstract}
We report the fabrication, properties, and bacteria-resistance of polyelectrolyte complex (PEC) coatings and free-standing films. Poly(4-styrenesulfonic acid), poly(diallyldimethyl-ammonium chloride), and salt were spin-coated into PEC films. After thermal annealing in a humid environment, highly transparent, mechanically strong, and chemically robust films were formed. Notably, we demonstrate that PEC coatings significantly reduce the attachment of Escherichia coli K12 without killing the micro-organisms. We suggest that forming bacteria-resistant surface coatings from commercially available polymers holds the potential for use across a wide range of applications including high-touch surfaces in medical settings.
\end{abstract}

\section{Graphical Abstract}

\section{Coacervate Films Repel Bacteria}

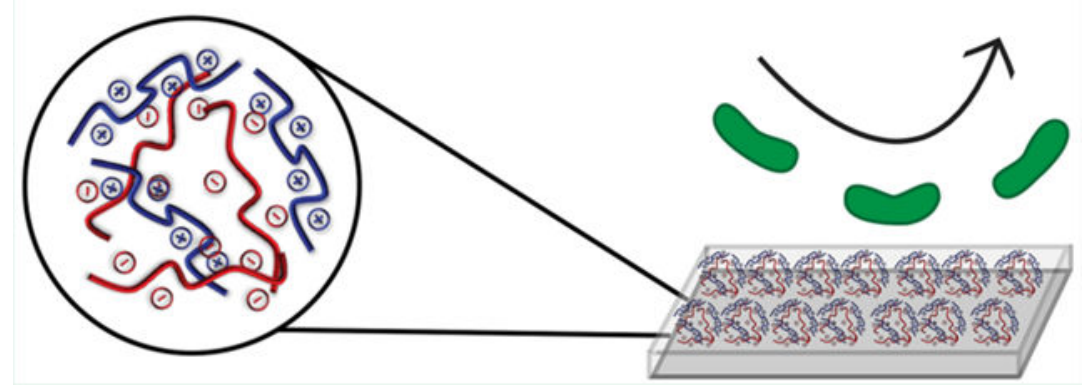

\section{Keywords}

antifouling; complex coacervation; Escherichia coli; polyelectrolyte; spin-coating

\footnotetext{
*Corresponding Authors: schiffman@ecs.umass.edu., perrys@engin.umass.edu.

$\dagger$ These authors contributed equally.

The authors declare no competing financial interest.

Supporting Information

The Supporting Information is available free of charge on the ACS Publications website at DOI: 10.1021/acsabm.9b00502.

Schematic of film preparation, transmittance, SAXS, WAXS, stress-strain curves, bovine serum albumin adsorption to films (PDF)
} 


\section{INTRODUCTION}

Inanimate surfaces in intensive care units (ICUs), such as bedrails, medical charts, and monitors, act as reservoirs for bacteria and cross-contamination, often leading to patient colonization and infections. ${ }^{1}$ Notably, approximately $60 \%$ of bacterial infections in ICUs are due to cross-transmission, necessitating effective and affordable means of preventing the initial attachment of bacteria. ${ }^{1}$ Typically, antifouling coatings feature hydrophilic polymers, such as poly(ethylene glycol) (PEG) or polymer zwitterions, ${ }^{2-4}$ which prevent bacterial adhesion by attracting water molecules to the surface, creating a hydration barrier to bacterial attachment. ${ }^{5,6}$ In particular for polymer zwitterion coatings, the close proximity of oppositely charged groups facilitates the formation of ionic bonds with water molecules, creating an aqueous layer that prevents the adsorption of organic and biological materials. ${ }^{5-8}$ While PEG and polymer zwitterion-based coatings are highly effective at reducing bacterial adhesion by at least $85 \%,{ }^{4,9-14}$ they often involve complex synthesis ${ }^{15-17}$ and substrate specific methods for surface immobilization. ${ }^{9,18-21}$ PEG is used extensively in commercial applications, ${ }^{22-25}$ but it can degrade in biological conditions, ${ }^{26-30}$ and PEG antibodies have been reported. Thus, developing alternative means of addressing biological fouling is needed. ${ }^{31-33}$

Polyelectrolyte complexes (PECs) form through the electrostatic and entropic interactions between oppositely charged polymers that lead to the release of bound counterions and the restructuring of the surrounding aqueous phase. ${ }^{34-36}$ The resulting complexes can undergo liquid-liquid phase separation (i.e., complex coacervation) or liquid-solid phase separation depending on the identity and length of the polymers, and the solution conditions, including the ionic strength and $\mathrm{pH}$ value. ${ }^{34,37,38}$ While PECs have been used in a range of applications including drug delivery, advanced adhesives, and food science, ${ }^{34,39,40}$ most of this work has been focused on liquid complex coacervates. The utility of solid PEC complexes has historically been limited as the strong electrostatic interactions that drive complexation prohibits traditional thermal or solvent processing methods. However, recent work has demonstrated that salt can be used to plasticize PECs to enable processing. ${ }^{41-48}$ To date, PECs have been extruded into single fibers, ${ }^{44,49}$ electrospun into fiber meshes, ${ }^{41,42}$ processed into free-standing microchambers, ${ }^{46}$ spin-coated into films, ${ }^{43}$ cast into films,,${ }^{45}$ and 3D-printed into material forms. ${ }^{50,51}$

Because PECs consist of oppositely charged polymers, we hypothesized that their positive and negative charges might mirror the functionality of polymer zwitterions, creating a barrier against bacterial attachment. ${ }^{3,5,6}$ Notably, layer-by-layer films show changes in wetting, indicating a possible hydration barrier. ${ }^{52-54}$ Previously, researchers have studied the antibacterial (killing) properties of silver nanoparticle-embedded polyelectrolyte layer-bylayer films, ${ }^{55}$ the flux recovery of layer-by-layer coated membranes, ${ }^{56}$ and their antifouling performance against diatom cells and barnacles. ${ }^{56,57}$ Another study described how ultracentrifugated compact PECs from chitosan and alginate resisted the adhesion of Staphylococcus aureus. ${ }^{58}$ While this result is encouraging, there is a benefit to developing alternative processing strategies that are less energy intensive and can be performed at scale to enable the use of PECs as antifouling coatings. 
Here, we report the fabrication of transparent, resilient, free-standing PEC films via spincoating and have investigated their application as bacteria-resistant coatings. PECs were formed using two low-cost, commercially available commodity polyelectrolytes, poly(4styrenesulfonic acid) and poly-(diallyldimethylammonium chloride) with potassium bromide $(\mathrm{KBr})$ salt. Spin-coated films were prepared using various postprocessing techniques, and their transparency, ultimate tensile strength, thickness, and resistance to the attachment of Escherichia coli K12 MG1655 were determined. Our strategy for the aqueous processing of low-cost polymers into antifouling coatings and free-standing films represents a crucial step toward potentially using these coatings to prevent microbial attachment in high-touch applications.

\section{MATERIALS AND METHODS}

\section{Materials and Chemicals.}

All compounds, unless otherwise noted, were used as received. Poly(4-styrenesulfonic acid, sodium salt) (PSS, AkzoNobel, VERSA TL130, 15 wt \%, ca. $70000 \mathrm{~g} / \mathrm{mol}, \mathrm{N} \approx 340$ ) was filtered using a $0.22 \mu \mathrm{m}$ pore size filter (EMD Millipore) prior to use. Poly(diallyldimethylammonium chloride) (PDADMAC, Hyperfloc CP 626, 20 wt \%, ca. 400 $000 \mathrm{~g} / \mathrm{mol}, \mathrm{N} \approx 2470$ ) was purchased from Hychem (Tampa, FL). Poly(2methacryloyloxyethyl phosphorylcholine) was purchased from Sigma-Aldrich and purified by washing with anhydrous diethyl ether. M9 minimal salts (M9 media), D-(+)-glucose, Luria-Bertani broth (LB), carbenicillin (BioReagent grade), dopamine hydrochloride, and propidium iodide (PI) were purchased from Sigma-Aldrich. Tris hydrochloride, Tris base, potassium bromide ( $\mathrm{KBr}$, ACS grade), hydrogen chloride ( $\mathrm{HCl}$, ACS grade), sodium hydroxide ( $\mathrm{NaOH}, \mathrm{ACS}$ grade), acetone (ACS reagent), and square glass coverslips (22 mm $\times 22 \mathrm{~mm}$ ) were purchased from Fisher Scientific (Hampton, NH). Barnstead Nanopure deionized (DI) water was obtained from a Milli-Q integral water purification system (resistivity of $18.2 \mathrm{M} \Omega \mathrm{cm}$, Millipore). The release layer LOR 5A (poly(dimethylglutarimide)) was purchased from Microchem (Westborough, MA), and silicon wafers (3 in., Type P, 100 orientation) were purchased from University Wafer (Boston, MA). RD6 developer (2.25-2.30\% tetramethylammonium hydroxide in water) was purchased from Futurrex (Franklin, NJ).

\section{Preparation of Complex Coacervates.}

Aqueous stock solutions of PSS and PDADMAC were prepared gravimetrically at a concentration of $0.5 \mathrm{M}$ on a monomer basis and adjusted to $\mathrm{pH} 7.2$ with a few drops of concentrated $\mathrm{HCl}$ or $\mathrm{NaOH}$. A stock solution of $4 \mathrm{M} \mathrm{KBr}$ was also prepared gravimetrically. Liquid complex coacervates were prepared at a total volume of $10 \mathrm{~mL}$ with a final concentration of $0.1 \mathrm{M}$ polymer, on a monomer basis, and a total $\mathrm{KBr}$ concentration of 1.6 $\mathrm{M}$ unless otherwise specified. PSS, $\mathrm{KBr}$, and DI water were added into a $15 \mathrm{~mL}$ centrifuge tube (Fisher Scientific) followed by PDADMAC. The system was then vortexed for $30 \mathrm{~s}$ and placed into a bath sonicator (Branson Ultrasonic Bath 2800, $40 \mathrm{kHz}$ transducer) for $15 \mathrm{~min}$ to facilitate mixing and equilibration. The resulting mixture was then centrifuged at 5000 rpm for $10 \mathrm{~min}$ (Sorvall ST 16R Centrifuge, Thermo Fisher Scientific) to coalesce the dense, 
polymer-rich coacervate phase. The polymer-poor supernatant phase was removed from the coacervate phase using a transfer pipet.

\section{Fabrication of Immobilized Coatings and Free-Standing PEC Films.}

Clean substrates (silicon wafers and glass coverslips) were prepared by rinsing with acetone, drying using nitrogen gas, and an oxygen plasma treatment (Harrick Plasma) for $1 \mathrm{~min}$. Next, $2 \mathrm{~mL}$ of isolated coacervate phase was uniformly dispensed and spin-coated (G3P-8, Specialty Coating System) onto the cleaned substrates. Varying spin rates from 1000 to 3000 $\mathrm{rpm}$ were evaluated using a $5 \mathrm{~s}$ ramp and a 1 min hold time for all samples. The resulting PEC coating was immediately immersed into a $23{ }^{\circ} \mathrm{C}$ DI water bath $(1 \mathrm{~L})$ for $10 \mathrm{~min}$ to remove salt and to allow the film to solidify. After the samples were removed from the water bath, excess water was removed by blotting with a Kimwipe (Kimtech). This process produced PEC coatings that were immobilized on a substrate.

For free-standing films, a release layer of LOR 5A (poly(dimethylglutarimide)) was first spin-coated onto the cleaned substrate at $3000 \mathrm{rpm}$ for $1 \mathrm{~min}$, followed by heating at $190{ }^{\circ} \mathrm{C}$ for 3 and $1 \mathrm{~min}$ of oxygen plasma treatment. After the coacervate was spin-coated using the procedure previously described, samples were immersed in RD6 developer (2.25-2.30\% tetramethylammonium hydroxide in water) for $5 \mathrm{~h}$ to dissolve the LOR 5A layer, which released the film. The released PEC film was then rinsed using DI water.

\section{Fabrication of Transparent and Opaque PEC Films.}

The transparency of immobilized and free-standing films was modulated post spin-coating. Transparent films were produced by annealing the water-saturated film at $120^{\circ} \mathrm{C}$ on a hot plate (Fisher Scientific) for $1 \mathrm{~min}$. Opaque films were obtained by either allowing the films to dry at $23{ }^{\circ} \mathrm{C}$ or by freezing the water-saturated film at $-80{ }^{\circ} \mathrm{C}$ overnight, followed by lyophilization (FreeZone, Labconco) (Figures 1 and S1).

\section{PEC Film Characterization.}

PEC films used for characterization tests were prepared from coacervates made in $1.6 \mathrm{M}$ $\mathrm{KBr}$ and spin-coated at $2000 \mathrm{rpm}$. The transparency of PEC films was measured by placing films immobilized on glass slides on top of a 96-well plate. The transmittance of the film was measured in triplicate using a Synergy plate reader (BioTek) from 310-750 nm. Film thickness measurements were determined using a stylus profilometer (Dektak 3, Veeco/ Sloan, Santa Barbara, CA) and a micrometer. Films attached to surfaces were first scratched using a razor blade to reveal the underlying substrate. Measurement scans were then run perpendicular to the scratches for a length of $2000 \mu \mathrm{m}$ at a duration of $20 \mathrm{~s}$ at $0.1 \mathrm{~mm} / \mathrm{s}$ using 500 point resolution. ${ }^{59}$ The thickness was determined to be the difference between the surface height and the lowest point of the scratch. Our profilometer data showed a clean substrate without roughness in the area of the scratch, which confirmed complete film removal. Film thicknesses were measured in triplicate. Free-standing films used for mechanical property characterization were measured using a Mitutoyo 293-330 digital micrometer by taking three measurements on each sample tested. 
Small- and wide-angle X-ray scattering (SAXS/WAXS) was used to characterize the internal structure of the PEC films using a Ganesha SAXS-Lab with Cu Ka X-ray source (1.54 ̊). Samples were prepared by folding one free-standing PEC film many times to achieve a total thickness of $0.5-1.6 \mathrm{~mm}$. Film samples were placed into the center opening of a metal washer and fixed using Kapton tape before being mounted on the X-ray beam. Exposure times of 10 and 3 min were used for SAXS and WAXS, respectively. Intensity data were normalized by the peak in the WAXS region during analysis. Contact angle measurements were performed using $4 \mu \mathrm{L}$ drops of DI water and glycerol using a home-built contact angle apparatus that was equipped with a Nikon D5100 digital camera with a $60 \mathrm{~mm}$ lens and 68 $\mathrm{mm}$ extension tube (Nikon, Melville, NY) ${ }^{60}$ At least nine measurements were acquired on each type of film, and the images were analyzed using ImageJ $1.51 j 8$ software (National Institutes of Health, Bethesda, MD).

Uniaxial mechanical testing was conducted using a Texture Analyzer (Texture Technologies) on free-standing PEC films $(1 \mathrm{~cm} \times 3 \mathrm{~cm})$. Ultimate tensile stress was calculated by dividing the measured maximum force by the cross-sectional area of the film. The cross-sectional area was the product of the film width times thickness (based on micrometer measurements). The PEC films were mounted on two clips using a silicone rubber sheet (McMaster-Carr) as the mounting medium and stretched at an extension rate of $\sim 3 \mathrm{~mm} / \mathrm{min}$ until failure. Tests were conducted on ten transparent films and six opaque films.

\section{Evaluation of Antibacterial and Antifouling Activity of PEC Films.}

The Gram-negative microorganism, Escherichia coli K12 MG1655 (E. coli, DSMZ, LeibnizInstitut, Germany) containing a green fluorescent protein (GFP) plasmid, was used in antibacterial and antifouling tests. E. coli (inoculated with $100 \mu \mathrm{g} / \mathrm{mL}$ carbenicillin) was cultured overnight in Luria-Bertani broth at $37^{\circ} \mathrm{C}$ to a concentration of $10^{8}$ cells $/ \mathrm{mL}$, washed twice, and resuspended in M9 media before use in either antibacterial or antifouling experiments.

For antibacterial tests, PEC films and polymer zwitterion controls were placed in separate wells of six-well polystyrene plates (Fisher Scientific), submerged in $5 \mathrm{~mL}$ of M9 media containing $10^{8} \mathrm{E}$. coli cells, and incubated without shaking at $37^{\circ} \mathrm{C}$ for $2 \mathrm{~h}$. Internal glass coverslips (cleaned by submerging in acetone at room temperature for $15 \mathrm{~min}$ followed by rinsing with autoclaved DI water three times, dried at $\sim 100{ }^{\circ} \mathrm{C}$ for $1 \mathrm{~h}$, and treated with UV/ ozone (UV/Ozone ProCleanerTM, Bioforce Nanosciences, Ames, IA) for $15 \mathrm{~min}$ ) were run in parallel for each experiment. Cells were stained in the dark with PI (60 $\mu \mathrm{M}$, excitation/ emission at $535 \mathrm{~nm} / 617 \mathrm{~nm}$ ), allowed to incubate for $15 \mathrm{~min},{ }^{61}$ and rinsed gently with M9 media before 15 random images were aquired, taken over three parallel replicates for three biological replicates using a Zeiss Microscope Axio Imager A2M (20× objective). GFP expressing E. coli were considered viable, while PI-stained E. coli were considered dead. ImageJ $1.51 j 8$ software was used to quantify the viable and dead cells, and the percentage of viable bacteria was determined using eq 1 :

$$
\text { Viable } E . \operatorname{coli}(\%)=\frac{\text { Viable } E \cdot \operatorname{col} i}{\text { Viable } E . \operatorname{coli}+\text { Dead } E . \operatorname{coli}} \times 100
$$


Antifouling tests were conducted on PEC films and polymer zwitterion controls, which were prepared based on a previously published method. ${ }^{9}$ Briefly, clean square glass coverslips (22 $\mathrm{mm} \times 22 \mathrm{~mm}$ ) were immersed in a solution of $10 \mathrm{mM}$ Tris buffer ( $\mathrm{pH} \mathrm{8.5),} \mathrm{poly(2-}$ methacryloyloxyethyl phosphorylcholine) (polymer zwitterion, $2 \mathrm{mg} / \mathrm{mL}$ ), and dopamine hydrochloride $(2 \mathrm{mg} / \mathrm{mL})$ for $6 \mathrm{~h}$ at $23{ }^{\circ} \mathrm{C}$. Excess polymer was removed via gentle rinsing with DI water before the samples were air-dried at $23{ }^{\circ} \mathrm{C}$. Antifouling tests were conducted by placing PEC films and polymer zwitterion controls in separate wells of six-well polystyrene plates and exposing them to $5 \mathrm{~mL}$ of $E$. coli $\left(10^{8}\right.$ cells $/ \mathrm{mL}$ of M9 media) without shaking for $24 \mathrm{~h}$ at $37^{\circ} \mathrm{C}$. Samples were gently rinsed using M9 media, and 15 randomly acquired images taken over three parallel replicates for three biological replicates were analyzed using ImageJ software to calculate the bacteria colony area coverage over the acquired $5504455 \mu \mathrm{m}^{2}$ area. Statistical significance was determined using a two-tailed, unpaired student $t$-test.

\section{RESULTS AND DISCUSSION}

\section{Characteristics of PEC Films.}

Spin-coating was used to successfully fabricate uniform coatings from complex coacervates formed from salt $(\mathrm{KBr})$ and two commercially purchased, strong polyelectrolytes, poly(4styrenesulfonic acid, sodium salt) (PSS) and poly(diallyldimethylammonium chloride) (PDADMAC), see Figures 1 and S1. Because of the hydrophilic nature of polyelectrolytes, substrates were first treated with oxygen plasma to facilitate good adhesion between the film and substrate and to avoid dewetting during the spin-coating process. This strategy allowed us to successfully prepare films on silicon wafers and glass slides in both the presence and absence of a polymeric release layer.

Freshly spin-coated films were transparent; however, they became opaque after being immersed in a water bath, which removed the salt (Figure 1). The amount of salt removed from the films was not directly quantified, but diffusion calculations based on results from Ghostine et al. suggest that rinsing of the films for a few seconds should be sufficient to remove nearly all of the salt. ${ }^{62}$ However, the increased opacity of the films suggests the formation of a nonuniform structure, potentially clusters of ions and water that are large enough to scatter light. Films removed from the water bath remained opaque if they were dried under ambient conditions or if they were lyophilized, similar to the work by Costa et al. ${ }^{45}$ Furthermore, once dried, the opacity of opaque films persisted after being immersed in water. Interestingly, films that were annealed at $120^{\circ} \mathrm{C}$ after salt removal immediately created a highly transparent film, and they maintained transparency despite subsequent humidity treatment. Similar film transparency could also be obtained by exposing a dried opaque film to hot steam or boiling water. Overall, annealing the films caused them to be very stable and more transparent than processing the films using steam or boiling water. We hypothesize that this transformation from opaque to transparent is the result of temperature and water-facilitated rearrangement of the polymer chains that allows for the relaxation of submicron clusters of water and ions that would otherwise scatter light. The literature describes a decrease in the gas permeability of analogous PEC films after thermal annealing in humid conditions. ${ }^{63}$ Transmittance experiments demonstrated that annealed PEC films 
had a similar transparency to that of glass slides in the wavelength range of $310-750 \mathrm{~nm}$, while air-dried films prepared using the same spin speed transmitted only $\sim 65 \%$ of the incident light, and lyophilized films were opaque (Figure S2).

PEC film thickness was investigated as a function of initial salt concentration, spin-coating speed, and post-treatment strategy (Figure 2). Film thickness decreased with increasing salt concentration and increasing spin speed, consistent with a report by Kelly and Schlenoff. ${ }^{43}$ These results were expected because the viscosity of the PSS/PDADMAC coacervate used for spin-coating decreases with increasing salt concentration, ${ }^{38,43,64,65}$ allowing for the formation of thinner films. Similarly, increasing the spin speed increases the centrifugal force, which subsequently thins the film. Comparing our results with those reported by Kelly and Schlenoff for the same polymer system, ${ }^{43}$ our films were significantly thinner (e.g., approximately $2.5 \mu \mathrm{m}$ vs $7.5 \mu \mathrm{m}$ for samples prepared at $1.7 \mathrm{M} \mathrm{KBr}$ and $2000 \mathrm{rpm}$ ). The difference in thickness is not surprising because the shorter polyelectrolytes used in our study lead to a coacervate solution that has a lower viscosity than the coacervate reported by Kelly and Schlenoff (i.e., $0.92 \mathrm{~Pa}$ s vs $1.42 \mathrm{~Pa} \mathrm{~s}$ ). ${ }^{64}$

Postprocessing also had the potential to influence film thickness. Lyophilized films were the thickest and potentially had a greater porosity, as suggested by their increased opacity, while transparent films were the thinnest. Specifically, at a salt concentration of $1.6 \mathrm{M}$ and a spin rate of $2000 \mathrm{rpm}$, lyophilized films were roughly six-times thicker than those prepared by thermal annealing and two-times thicker than those prepared by air drying. Analysis via SAXS and WAXS suggests that the postprocessing method did not significantly affect the molecular-level structure of the films (Figure S3). However, the scattering signal for the opaque films was consistently higher than the signal of the transparent films at low q, suggesting that differences in the structure may exist at larger length scales (i.e., smaller $q$ values) than what was accessible in the measurement. This possibility of larger aggregates agrees with the observed differences in transparency and the potential for submicron scale clusters.

DI water and glycerol are two liquids that have different surface tensions and viscosities. Therefore, we conducted contact angle experiments using these two different liquids to determine the wettability of the transparent and opaque PEC films, see Figure 3 . To be considered hydrophilic, a material typically has a contact angle less than $90^{\circ}$; notably, hydrophilic surfaces have been reported to have strong antifouling properties including an improved resistance to adhesion by bacteria. ${ }^{5,6}$ Polymer zwitterion films with known hydrophilicity were used as controls. ${ }^{9}$ Water contact angles for transparent, opaque air-dried, and opaque lyophilized PEC films were $33.9 \pm 2.6^{\circ}, 31.2 \pm 2.7^{\circ}$, and $28.9 \pm 4.2^{\circ}$, respectively, which are all statistically higher than the polymer zwitterion films (14.2 $\pm 2.7^{\circ}$ ). Similarly, the glycerol contact angles for transparent, opaque air-dried, and opaque lyophilized PEC films were $50.7 \pm 4.2^{\circ}, 48.4 \pm 4.3^{\circ}$, and $40.1 \pm 2.7^{\circ}$, respectively, again, statistically greater than the glycerol contact angle of the polymer zwitterion films (32.8 $\pm 3.7^{\circ}$ ). While the polymer zwitterion control was more hydrophilic than the PEC films, the PEC films are still considered hydrophilic. 
PEC materials have long been considered intractable for processing. Because of the electrostatic interactions driving their self-assembly, PEC materials have excellent resistance against dissolution by organic solvents. ${ }^{39} \mathrm{We}$ have previously demonstrated this resistance for fiber mats that were electrospun using the same PEC system used in this work. ${ }^{41}$ Additionally, the strong polyelectrolytes used in this study are resistant to variations in $\mathrm{pH}$ value. As a result, we expect that our films would show similar durability as the previously demonstrated PEC materials including our fibers. Most interestingly, while high concentrations of salt have been shown to plasticize PEC materials, our thermally annealed PEC films were unaffected by immersion in a $4 \mathrm{M} \mathrm{KBr}$ solution, even after one month. This result suggests the possibility of using thermal treatment to further enhance the stability of a wide range of PEC-based materials.

In addition to the transparency, wetting, and physical stability of our films, mechanical properties are critical for enabling the use of high-performance coatings in real world settings. To this end, we performed uniaxial tensile tests on free-standing transparent and opaque PEC films. The transparent PEC films exhibited an ultimate tensile strength that was remarkably 4.2-fold greater than that of the opaque air-dried PEC films and stronger than that of other reported PEC films (Figures 4 and S4). The average Young's modulus of the transparent films was found to be $29.07 \pm 8.2 \mathrm{MPa}$, whereas a value of $6.01 \pm 0.9 \mathrm{MPa}$ was obtained for the opaque air-dried films. As evident from our reported standard deviations and Figure S4, which displays the stress-strain curves from which the mechanical properties were calculated, the transparent films exhibited more sample-to-sample variation than the opaque air-dried films. All free-standing PEC films exhibited a brittle fracture toward the middle of the films with a correspondingly low strain value of $\sim 2 \%$, and previous reports also suggested that PECs have a low strain at break of $\sim 5 \% .{ }^{43}$ In general, our data are consistent with other reports that demonstrate increased mechanical properties after annealing, ${ }^{44,66,67}$ and we attribute the increase in ultimate tensile stress to an increase in the concentration of ionic bonds in the material as well as a removal of stress-concentrating defects associated with the submicron clusters associated with the opacity of the untreated material.

To determine if the PEC films inactivated microorganisms, we conducted a live/dead assay using E. coli (Figure 5). Transparent PEC films, polymer zwitterion control films, and glass had a negligible effect on $E$. coli viability, with statistically equivalent viability of at least $90 \%$, indicating no killing. While cationic polymers, including the one we used to form our coacervate films, PDADMAC, have antibacterial properties, ${ }^{61}$ the ion pairing of equimolar concentrations of PSS with PDADMAC eliminated these antibacterial properties. Our findings are consistent with experiments conducted on ultracentrifugated compact polyelectrolyte complexes formed from chitosan and alginate, which also demonstrated negligible killing of Staphylococcus aureus. ${ }^{58}$ Charge-neutral polyelectrolyte complexes do not exhibit the antibacterial properties of their cationic polyelectrolyte components. Liquid complex coacervates have also been reported to be biocompatible with mammalian cells. 68,69

The antifouling properties (how many bacteria adhere) to the PEC films were assessed using E. coli cultured in minimal media, see Figure 6. In comparison to control glass slides, PEC 
films reduced the adhesion of E. coli by over $75 \%$, from $\sim 11 \%$ for glass to $\sim 2.5 \%$ for transparent and air-dried PEC films. ${ }^{70}$ Furthermore, we observed a statistically equivalent performance between the PEC films and the polymer zwitterion coatings. While quantification was not provided for bacterial adhesion on ultracentrifuged compact PECs formed from chitosan and alginate, qualitative comparisons suggest comparable performance. ${ }^{58}$ Our results demonstrate that PEC films are extremely antifouling to the microorganism E. coli, regardless of their transparency, and are equivalent to the polymer zwitterion films.

The hydrophilicity and strong microbial resistance properties of the PEC films are likely due to the proximity of positive and negative charges that bind PEC materials together. Sum frequency generation (SFG) spectroscopy measurements on zwitterionic and a 1:1 mixed charge polymer surface demonstrated strong levels of surface hydration and thus antifouling character. ${ }^{3,5-8}$ Furthermore, the use of polyelectrolyte complexation as a processing strategy allows for the formation of stable, water-insoluble films without the need for organic solvents. It should be noted that one limitation of our current PEC films is that they did not prevent the attachment of the protein, bovine serum albumin, Figure S5. While beyond the scope of this study, it is possible that by incorporating small concentrations of PEG or polymer zwitterions into the PEC films that their protein fouling resistance would be enhanced. The results from this study indicate that PEC films have the potential to offer an economic means of decreasing bacterial attachment.

\section{CONCLUSION}

We have established a straightforward method to fabricate robust, transparent, ultralow bacterial-fouling PEC films via spin-coating of complex coacervates, followed by thermal annealing in a humid environment. The ability to create such resilient materials using wateronly processing allows for environmentally friendly processing and can facilitate the use of PEC-based coatings in a wide range of applications. Notably, the absence of any organic solvents or cross-linking agents allows for the safety and biocompatibility necessary for implementing antifouling coatings. This work paves the way for future exploration of novel PEC-based materials including their use in biomedical applications as coatings for inanimate surfaces in hospitals and noninvasive biomedical equipment.

\section{Supplementary Material}

Refer to Web version on PubMed Central for supplementary material.

\section{ACKNOWLEDGMENTS}

We acknowledge the support of the National Science Foundation (NSF CMMI-1727660). I.S.K. acknowledges the support of the National Science Foundation NRT-SMLS program (DGE-1545399). M.H. was supported by National Research Service Award No. T32 GM008515 from the National Institutes of Health. We would also like to thank Dr. Todd Emrick, Xiangxi Meng, and Dr. Benjamin Yavitt for their helpful conversations. 


\section{REFERENCES}

(1). Agodi A; Barchitta M; Cipresso R; Giaquinta L; Romeo MA; Denaro C Pseudomonas Aeruginosa Carriage, Colonization, and Infection in ICU Patients. Intensive Care Med. 2007, 33 (7), 11551161. [PubMed: 17503016]

(2). Kurtz IS; Schiffman JD Current and Emerging Approaches to Engineer Antibacterial and Antifouling Electrospun Nanofibers. Materials 2018, 11 (7), 1059.

(3). Leng C; Huang H; Zhang K; Hung HC; Xu Y; Li Y; Jiang S; Chen Z Effect of Surface Hydration on Antifouling Properties of Mixed Charged Polymers. Langmuir 2018, 34 (22), 6538-6545. [PubMed: 29733605]

(4). Schlenoff JB Zwitteration: Coating Surfaces with Zwitterionic Functionality to Reduce Nonspecific Adsorption. Langmuir 2014, 30(32), 9625-9636. [PubMed: 24754399]

(5). Hower JC; Bernards MT; Chen S; Tsao HK; Sheng YJ; Jiang S Hydration of "Nonfouling" Functional Groups. J. Phys. Chem. B 2009, 113 (1), 197-201. [PubMed: 19072165]

(6). White A; Jiang S Local and Bulk Hydration of Zwitterionic Glycine and Its Analogues through Molecular Simulations. J. Phys. Chem. B 2011, 115 (4), 660-667. [PubMed: 21174438]

(7). Evers LH; Bhavsar D; Mailänder P The Biology of Burn Injury. Exp. Dermatol 2010, 19 (9), $777-$ 783. [PubMed: 20629737]

(8). Tegoulia VA; Rao W; Kalambur AT; Rabolt JF; Cooper SL Surface Properties, Fibrinogen Adsorption, and Cellular Interactions of a Novel Phosphorylcholine-Containing Self-Assembled Monolayer on Gold. Langmuir 2001, 17 (14), 4396-4404.

(9). Chang C-C; Kolewe KW; Li Y; Kosif I; Freeman BD; Carter KR; Schiffman JD; Emrick T Underwater Super-oleophobic Surfaces Prepared from Polymer Zwitterion/Dopamine Composite Coatings. Adv. Mater. Interfaces 2016, 3 (6), 1500521. [PubMed: 27774375]

(10). Banerjee I; Pangule RC; Kane RS Antifouling Coatings: Recent Developments in the Design of Surfaces That Prevent Fouling by Proteins, Bacteria, and Marine Organisms. Adv. Mater 2011, 23(6), 690-718. [PubMed: 20886559]

(11). Hucknall A; Rangarajan S; Chilkoti A In Pursuit of Zero: Polymer Brushes That Resist the Adsorption of Proteins. Adv. Mater 2009, 21 (23), 2441-2446.

(12). Mi L; Jiang S Integrated Antimicrobial and Nonfouling Zwitterionic Polymers. Angew. Chem., Int. Ed 2014, 53 (7), 1746-1754.

(13). Li G; Cheng G; Xue H; Chen S; Zhang F; Jiang S Ultra Low Fouling Zwitterionic Polymers with a Biomimetic Adhesive Group. Biomaterials 2008, 29 (35), 4592-4597. [PubMed: 18819708]

(14). Keefe AJ; Jiang S Poly(Zwitterionic)Protein Conjugates Offer Increased Stability without Sacrificing Binding Affinity or Bioactivity. Nat. Chem 2012, 4 (1), 59-63.

(15). Kyomoto M; Moro T; Takatori Y; Kawaguchi H; Nakamura K; Ishihara K Self-Initiated Surface Grafting with Poly(2-Methacryloyloxyethyl Phosphorylcholine) on Poly(Ether-Ether-Ketone). Biomaterials 2010, 31 (6), 1017-1024. [PubMed: 19906420]

(16). Kuang J; Messersmith PB Universal Surface-Initiated Polymerization of Antifouling Zwitterionic Brushes Using a Mussel-Mimetic Peptide Initiator. Langmuir 2012, 28 (18), 7258-7266. [PubMed: 22506651]

(17). Zhang Z; Chao T; Chen S; Jiang S Superlow Fouling Sulfobetaine and Carboxybetaine Polymers on Glass Slides. Langmuir 2006, 22 (24), 10072-10077. [PubMed: 17107002]

(18). Chang Y; Shih YJ; Lai CJ; Kung HH; Jiang S Blood-Inert Surfaces via Ion-Pair Anchoring of Zwitterionic Copolymer Brushes in Human Whole Blood. Adv. Funct. Mater 2013, 23 (9), 11001110.

(19). Futamura K; Matsuno R; Konno T; Takai M; Ishihara K Rapid Development of Hydrophilicity and Protein Adsorption Resistance by Polymer Surfaces Bearing Phosphorylcholine and Naphthalene Groups. Langmuir 2008, 24 (18), 10340-10344. [PubMed: 18698868]

(20). Seo JH; Matsuno R; Takai M; Ishihara K Cell Adhesion on Phase-Separated Surface of Block Copolymer Composed of Poly(2-Methacryloyloxyethyl Phosphorylcholine) and Poly(Dimethylsiloxane). Biomaterials 2009, 30 (29), 5330-5340. [PubMed: 19592090] 
(21). Ueda T; Oshida H; Kurita K; Ishihara K; Nakabayashi N Preparation of 2-Methacryloyloxyethyl Phosphorylcholine Copolymers with Alkyl Methacrylates and Their Blood Compatibility. Polym. J 1992, 24 (11), 1259-1269.

(22). D'souza AA; Shegokar R Polyethylene Glycol (PEG): A Versatile Polymer for Pharmaceutical Applications. Expert Opin. Drug Delivery 2016, 13 (9), 1257-1275.

(23). Abuchowski A; McCoy JR; Palczuk NC; van Es T; Davis FF Effect of Covalent Attachment of Polyethylene Glycol on Immunogenicity and Circulating Life of Bovine Liver Catalase. J. Biol. Chem 1977, 252 (11), 3582-3586. [PubMed: 16907]

(24). Knop K; Hoogenboom R; Fischer D; Schubert US Poly(Ethylene Glycol) in Drug Delivery: Pros and Cons as Well as Potential Alternatives. Angew. Chem., Int. Ed 2010, 49 (36), 6288-6308.

(25). Kolate A; Baradia D; Patil S; Vhora I; Kore G; Misra A PEG - A Versatile Conjugating Ligand for Drugs and Drug Delivery Systems. J. Controlled Release 2014, 192 (28), 67-81.

(26). Ulbricht J; Jordan R; Luxenhofer R On the Biodegradability of Polyethylene Glycol, Polypeptoids and Poly(2-Oxazoline)S. Biomaterials 2014, 35 (17), 4848-4861. [PubMed: 24651032]

(27). McGary CW Degradation of Poly(Ethylene Oxide). J. Polym. Sci 1960, 46 (147), 51-57.

(28). Han S; Kim C; Kwon D Thermal/Oxidative Degradation and Stabilization of Polyethylene Glycol. Polymer 1997, 38 (2), 317-323.

(29). Branch DW; Wheeler BC; Brewer GJ; Leckband DE Long-Term Stability of Grafted Polyethylene Glycol Surfaces for Use with Microstamped Substrates in Neuronal Cell Culture. Biomaterials 2001, 22 (10), 1035-1047. [PubMed: 11352085]

(30). Pidhatika B; Rodenstein M; Chen Y; Rakhmatullina E; Mühlebach A; Acikgöz C; Textor M; Konradi R Comparative Stability Studies of Poly(2-Methyl-2-Oxazoline) and Poly(Ethylene Glycol) Brush Coatings. Biointerphases 2012, 7 (1), 1-15. [PubMed: 22589044]

(31). Yang Q; Lai SK Anti-PEG Immunity: Emergence, Characteristics, and Unaddressed Questions. Wiley Interdiscip. Rev. Nanomedicine Nanobiotechnology 2015, 7 (5), 655-677. [PubMed: 25707913]

(32). Zhang P; Sun F; Liu S; Jiang S Anti-PEG Antibodies in the Clinic: Current Issues and beyond PEGylation. J. Controlled Release 2016, 244, 184-193.

(33). Yang Q; Jacobs TM; McCallen JD; Moore DT; Huckaby JT; Edelstein JN; Lai SK Analysis of Pre-Existing IgG and IgM Antibodies against Polyethylene Glycol (PEG) in the General Population. Anal. Chem 2016, 88 (23), 11804-11812. [PubMed: 27804292]

(34). Blocher WC; Perry SL Complex Coacervate-Based Materials for Biomedicine. Wiley Interdiscip. Rev. Nanomedicine Nanobiotechnology 2017, 9 (4), No. e1442.

(35). Sing CE Development of the Modern Theory of Polymeric Complex Coacervation. Adv. Colloid Interface Sci 2017, 239, 2-16. [PubMed: 27161661]

(36). Priftis D; Laugel N; Tirrell M Thermodynamic Characterization of Polypeptide Complex Coacervation. Langmuir 2012, 28(45), 15947-15957. [PubMed: 23083137]

(37). Perry SL; Li Y; Priftis D; Leon L; Tirrell M The Effect of Salt on the Complex Coacervation of Vinyl Polyelectrolytes. Polymers 2014, 6 (6), 1756-1772.

(38). Wang Q; Schlenoff JB The Polyelectrolyte Complex/Coacervate Continuum. Macromolecules 2014, 47 (9), 3108-3116.

(39). Michaels AS Polyelectrolyte Complexes. Ind. Eng. Chem 1965, 57 (10), 32-40.

(40). Kalantar TH; Tucker CJ; Zalusky AS; Boomgaard TA; Wilson BE; Ladika M; Jordan SL; Li WK; Zhang X; Goh CG High Throughput Workflow for Coacervate Formation and Characterization in Shampoo Systems. J. Cosmet. Sci 2007, 58 (4), 375-383. [PubMed: 17728938]

(41). Meng X; Perry SL; Schiffman JD Complex Coacervation: Chemically Stable Fibers Electrospun from Aqueous Polyelectrolyte Solutions. ACS Macro Lett. 2017, 6 (5), 505-511.

(42). Meng X; Schiffman JD; Perry SL Electrospinning Cargo-Containing Polyelectrolyte Complex Fibers: Correlating Molecular Interactions to Complex Coacervate Phase Behavior and Fiber Formation. Macromolecules 2018, 51 (21), 8821-8832.

(43). Kelly KD; Schlenoff JB Spin-Coated Polyelectrolyte Coacervate Films. ACS Appl. Mater. Interfaces 2015, 7 (25), 13980-13986. 
(44). Shamoun RF; Reisch A; Schlenoff JB Extruded Saloplastic Polyelectrolyte Complexes. Adv. Funct. Mater 2012, 22 (9), 1923-1931.

(45). Costa RR; Costa AMS; Caridade SG; Mano JF Compact Saloplastic Membranes of Natural Polysaccharides for Soft Tissue Engineering. Chem. Mater 2015, 27 (21), 7490-7502.

(46). Gai M; Frueh J; Kudryavtseva VL; Mao R; Kiryukhin MV; Sukhorukov GB Patterned Microstructure Fabrication: Polyelectrolyte Complexes vs Polyelectrolyte Multilayers. Sci. Rep 2016, 6, 37000. [PubMed: 27830831]

(47). Hariri HH; Schlenoff JB Saloplastic Macroporous Polyelectrolyte Complexes: Cartilage Mimics. Macromolecules 2010, 43 (20), 8656-8663. [PubMed: 21132107]

(48). Porcel CH; Schlenoff JB Compact Polyelectrolyte Complexes: "Saloplastic" Candidates for Biomaterials. Biomacromolecules 2009, 10 (11), 2968-2975. [PubMed: 19835412]

(49). Wang Q; Schlenoff JB Tough Strained Fibers of a Polyelectrolyte Complex: Pretensioned Polymers. RSC Adv. 2014, 4(87), 46675-46679.

(50). Liu Q; Li Q; Xu S; Zheng Q; Cao X Preparation and Properties of 3D Printed Alginate-Chitosan Polyion Complex Hydrogels for Tissue Engineering. Polymers 2018, 10 (6), 664.

(51). Zhu F; Cheng L; Yin J; Wu ZL; Qian J; Fu J; Zheng Q 3D Printing of Ultratough Polyion Complex Hydrogels. ACS Appl. Mater. Interfaces 2016, 8 (45), 31304-31310. [PubMed: 27779379]

(52). Diamanti E; Muzzio N; Gregurec D; Irigoyen J; Pasquale M; Azzaroni O; Brinkmann M; Moya SE Impact of Thermal Annealing on Wettability and Antifouling Characteristics of Alginate Poly-L-Lysine Polyelectrolyte Multilayer Films. Colloids Surf., B 2016, 145, 328-337.

(53). Kolasińska M; Warszyński P The Effect of Nature of Polyions and Treatment after Deposition on Wetting Characteristics of Polyelectrolyte Multilayers. Appl. Surf. Sci 2005, 252 (3), 759-765.

(54). Elzbieciak M; Kolasinska M; Warszynski P Characteristics of Polyelectrolyte Multilayers: The Effect of Polyion Charge on Thickness and Wetting Properties. Colloids Surf., A 2008, 321 (13), 258-261.

(55). Francesko A; Ivanova K; Hoyo J; Pérez-Rafael S; Petkova P; Fernandes MM; Heinze T; Mendoza E; Tzanov T Bottom-up Layer-by-Layer Assembling of Antibacterial Freestanding Nanobiocomposite Films. Biomacromolecules 2018, 19 (9), 3628-3636. [PubMed: 30052024]

(56). Tripathi BP; Dubey NC; Stamm M Functional Polyelectrolyte Multilayer Membranes for Water Purification Applications. J. Hazard. Mater 2013, 252-253, 401-412. [PubMed: 23611806]

(57). Zhu X; Jańczewski D; Lee SSC; Teo SLM; Vancso GJ Cross-Linked Polyelectrolyte Multilayers for Marine Antifouling Applications. ACS Appl. Mater. Interfaces 2013, 5 (13), 5961-5968. [PubMed: 23781913]

(58). Phoeung T; Spanedda MV; Roger E; Heurtault B; Fournel S; Reisch A; Mutschler A; PerrinSchmitt F; Hemmerlé J; Colin D; Rawiso F; Boulmedais F; Schaaf P; Lavalle P; Frisch B Alginate/Chitosan Compact Polyelectrolyte Complexes: A Cell and Bacterial Repellent Material. Chem. Mater 2017, 29 (24), 10418-10425.

(59). Rieger KA; Eagan NM; Schiffman JD Encapsulation of Cinnamaldehyde into Nanostructured Chitosan Films. J. Appl. Polym. Sci 2015, 132 (13), 1. [PubMed: 25866416]

(60). Ishino C; Okumura K; Quéré D Wetting Transitions on Rough Surfaces. Europhys. Lett 2004, 68 (3), 419-425.

(61). Rieger KA; Porter M; Schiffman JD Polyelectrolyte-Functionalized Nanofiber Mats Control the Collection and Inactivation of Escherichia Coli. Materials 2016, 9 (4), 297.

(62). Ghostine RA; Markarian MZ; Schlenoff JB Asymmetric Growth in Polyelectrolyte Multilayers. J. Am. Chem. Soc 2013, 135(20), 7636-7646. [PubMed: 23672490]

(63). Haile M; Henderson R; Grunlan JC; Sarwar O; Smith R Polyelectrolyte Coacervates Deposited as High Gas Barrier Thin Films. Macromol. Rapid Commun 2017, 38 (1), 1600594.

(64). Liu Y; Momani B; Winter HH; Perry SL Rheological Characterization of Liquid-to-Solid Transitions in Bulk Polyelectrolyte Complexes. Soft Matter 2017, 13 (40), 7332-7340. [PubMed: 28951897]

(65). Spruijt E; Cohen Stuart MA; Van Der Gucht J Linear Viscoelasticity of Polyelectrolyte Complex Coacervates. Macromolecules 2013, 46 (4), 1633-1641. 
(66). Wang XS; Ji YL; Zheng PY; An QF; Zhao Q; Lee KR; Qian JW; Gao CJ Engineering Novel Polyelectrolyte Complex Membranes with Improved Mechanical Properties and Separation Performance. J. Mater. Chem. A 2015, 3 (14), 7296-7303.

(67). Cai N; Han C; Luo X; Liu S; Yu F Rapidly and Effectively Improving the Mechanical Properties of Polyelectrolyte Complex Nanofibers through Microwave Treatment. Adv. Eng. Mater 2017, 19(1), 1600483.

(68). Black KA; Priftis D; Perry SL; Yip J; Byun WY; Tirrell M Protein Encapsulation via Polypeptide Complex Coacervation. ACS Macro Lett. 2014, 3 (10), 1088-1091.

(69). Giannotti MI; Abasolo I; Oliva M; Andrade F; García-Aranda N; Melgarejo M; Pulido D; Corchero JL; Fernández Y; Schwartz S; et al. Highly Versatile Polyelectrolyte Complexes for Improving the Enzyme Replacement Therapy of Lysosomal Storage Disorders. ACS Appl. Mater. Interfaces 2016, 8 (39), 25741-25752. [PubMed: 27610822]

(70). Elder RM; Emrick T; Jayaraman A Understanding the Effect of Polylysine Architecture on DNA Binding Using Molecular Dynamics Simulations. Biomacromolecules 2011, 12 (11), 3870-3879. [PubMed: 21961938] 
(a)

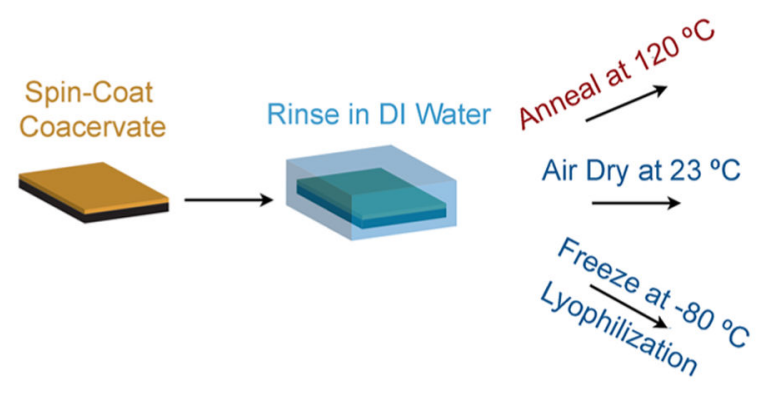

(b)

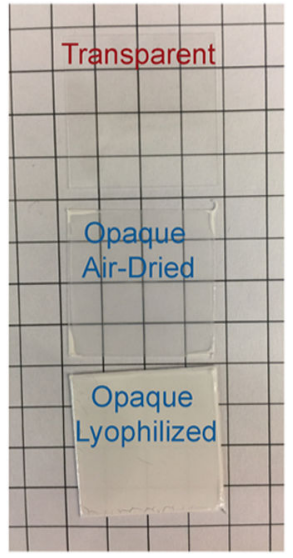

Figure 1.

(a) General fabrication schematic and (b) photographs of transparent, opaque air-dried, and opaque lyophilized PEC films. For a schematic of the full process, see Figure S1. 


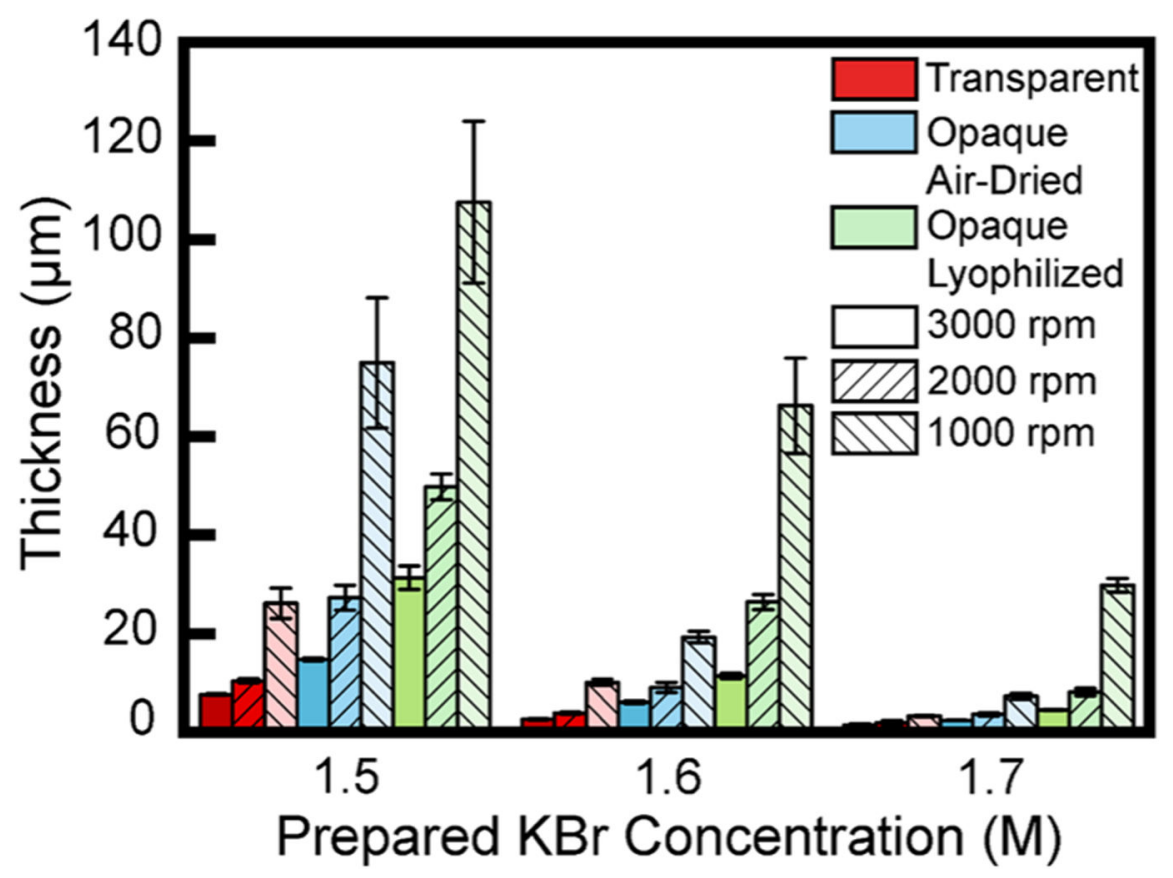

Figure 2.

Thickness of immobilized transparent, opaque air-dried, and opaque lyophilized PEC films as a function of the as-prepared salt concentration and spin-coating speed. Error bars denote standard deviation. 


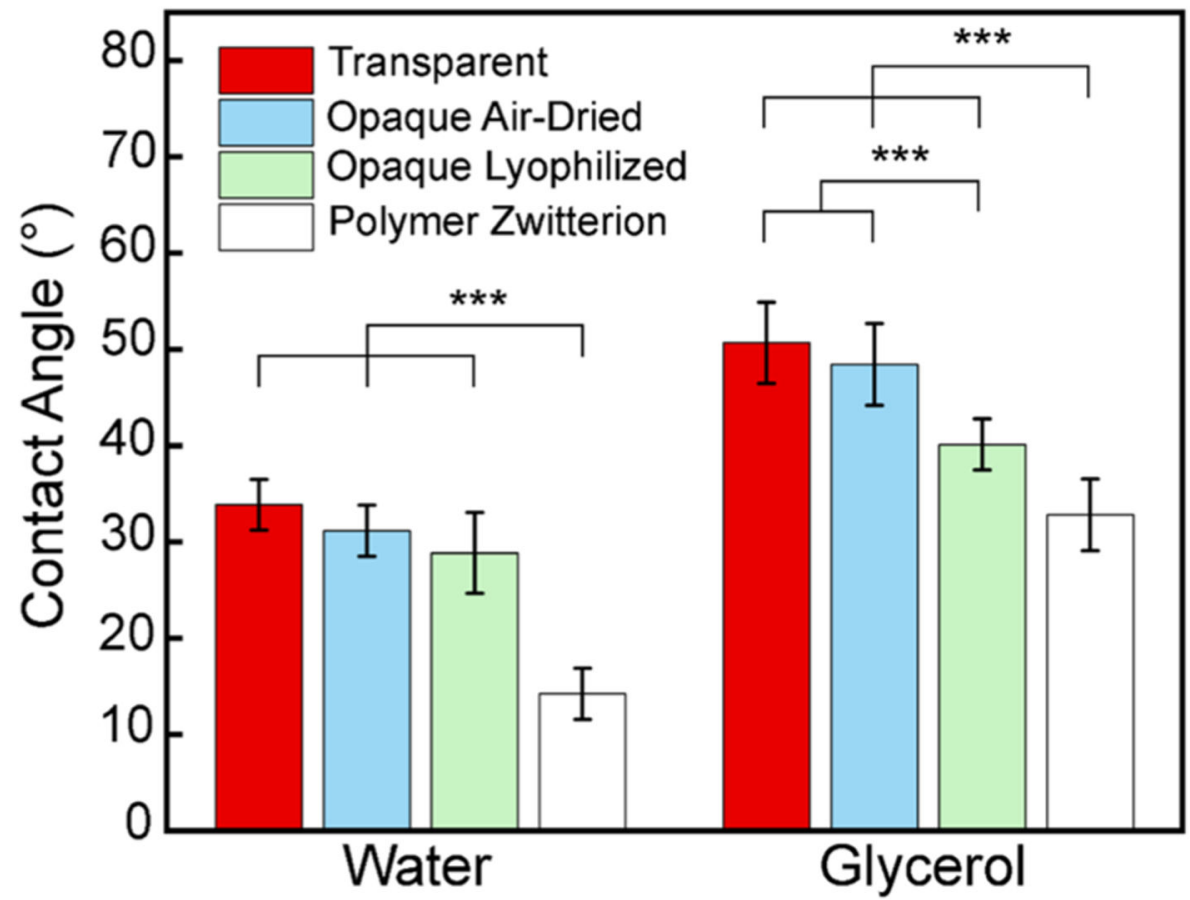

Figure 3.

DI water and glycerol contact angle measurements of transparent and opaque PEC films as well as polymer zwitterion controls. PEC films were prepared using PSS/PDADMAC with 1.6 M KBr and spin-coated at $2000 \mathrm{rpm}$. Three asterisks (***) denote $99.9 \%$ significance between samples. Error bars denote standard deviation. 


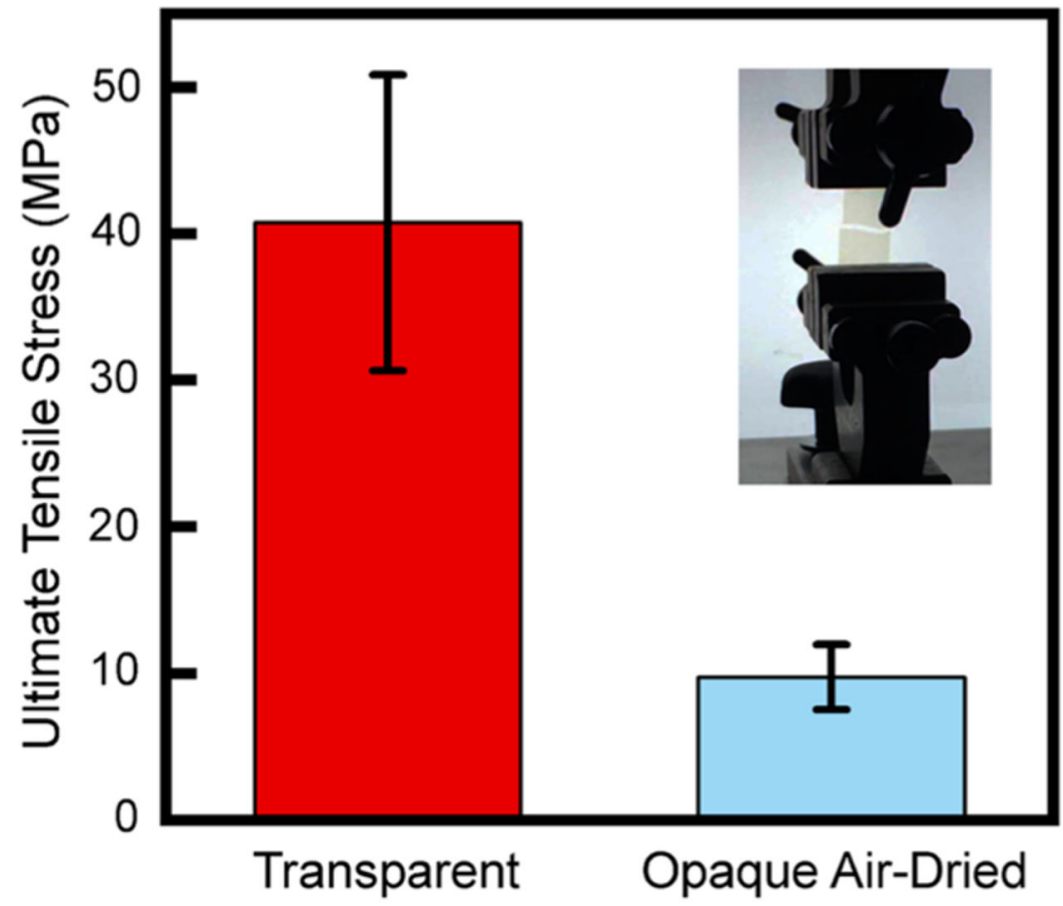

Figure 4.

Ultimate tensile stress of transparent and opaque PEC films. Inset photo displays the experimental setup. PEC films were prepared using PSS/PDADMAC with 1.6 M KBr and spin-coated at $2000 \mathrm{rpm}$. Error bars denote standard deviation. Stress-strain curves acquired on the transparent and opaque PEC films are provided in Figure S4. 
(a)

Figure 5.

(a) E. coli viability and (b) representative micrographs of E. coli after a 2 h incubation with transparent PEC films as well as polymer zwitterion and glass controls. Errors bars denote standard error, and n.s. indicates no statistical significance.
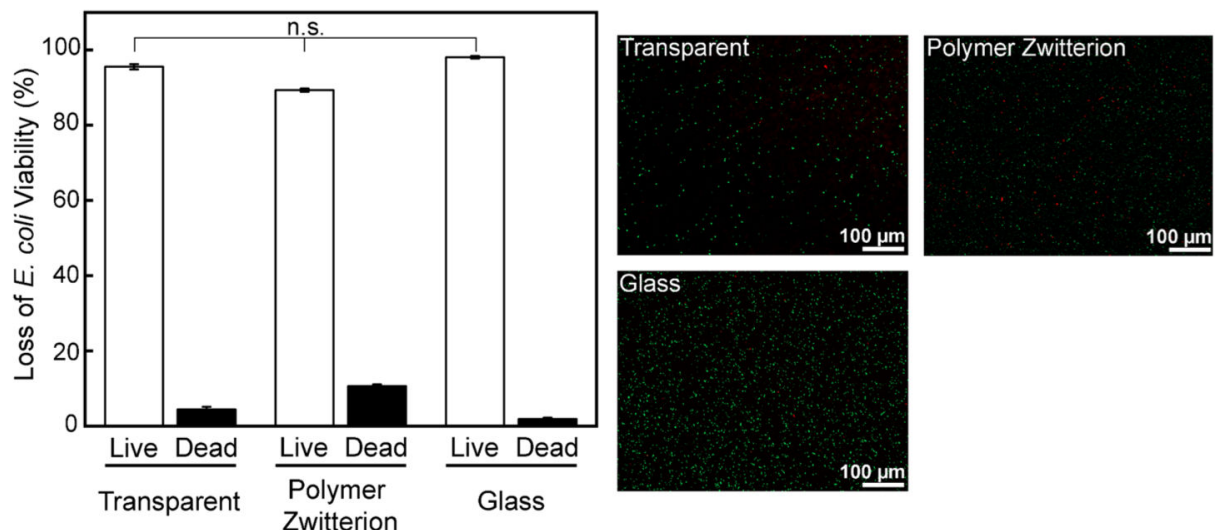
(a)

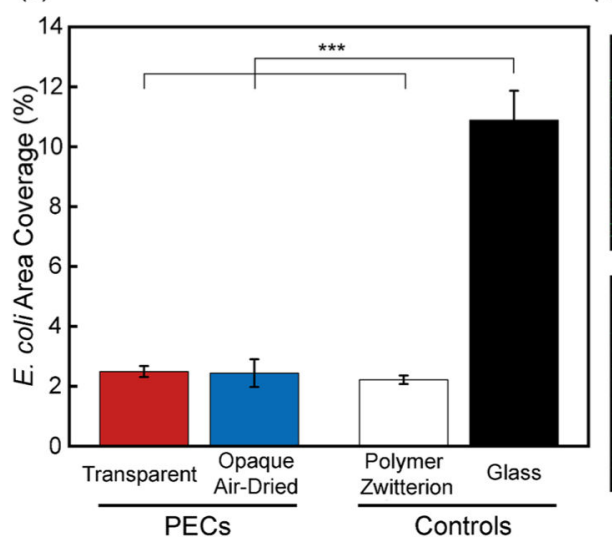

(b)

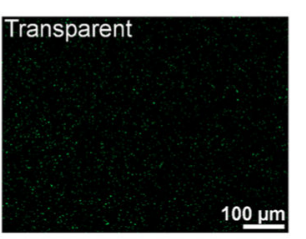

Opaque Air-Driéd
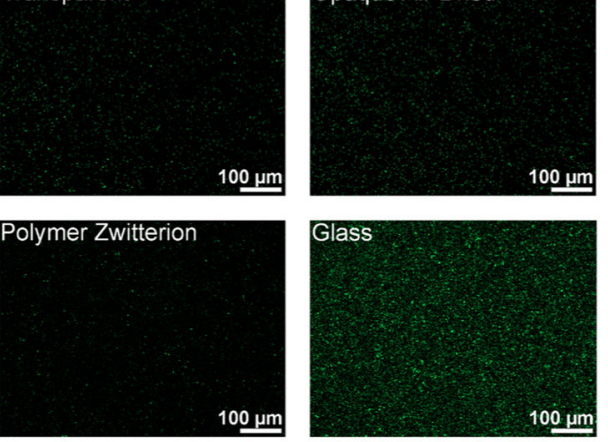

Figure 6.

(a) Antifouling activity and (b) representative micrographs of transparent and opaque PEC films as well as polymer zwitterion and glass controls after a $24 \mathrm{~h}$ incubation with $E$. coli. Three asterisks $(* * *)$ denote $99.9 \%$ significance between samples. Error bars denote standard error. 